\title{
Argon test cell Coaxial Cable Feed Through Connector Testing
}

\author{
R.T. Krasa
}

10/31/88

D-Zero Engineering Note \#3740.000.EN-190

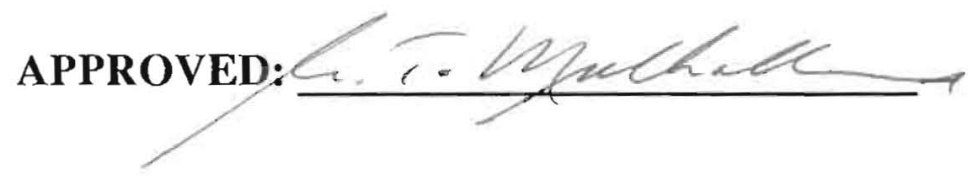




\section{Coaxial Cable Feedthrough}

Within each cryostat their will exist three Argon

Monitoring Boxes. Located at different elevations these boxes are responsible for measuring the relative "quality" of the Argon as far as it's ability to transfer electrons without recombination. Each box requires three miniature coaxial cables to provide a signal to the outside world.

These cables are shielded in groups of three. Each cryostat requires three of these grouped cables which must pass through some form of feedthrough located in the Instrumentation Box. For reasons of minimizing crosstalk and signal loss it is best to provide uninterrupted coaxial service between the receiving device and the monitor boxes.

In an attempt to provide such service Jerry Blazey obtained a connector whose manufacturer promised would provide uninterupted coaxial service. Results of cold shock and leakage tests preformed on this feedthrough comprise the remainder of this note. Under normal operating conditions this feedthrough would never reach temperatures as low as those used for these cold shock tests. 


\section{Coaxial Cable Feed through \\ Coaxial Cable Feedthrough Test Results}

Initial leakage rate before testing

4 x 10E-9 std. cc He/sec.

Cold Shock:

-LN2 was poured onto the wires approximately one inch away from the epoxy. Eventually(90sec.) the epoxy did reach LN2 temperature.

while cold

Leakage Rate.

warm-up:

$20 \mathrm{~min}$.

4 hrs.

4 x 10E-9 std. cc He/sec.

$18 \mathrm{hrs}$.

$3 \times 10 \mathrm{E}-7$ std. $\mathrm{cc} \mathrm{He} / \mathrm{sec}$.

$1 \times 10 \mathrm{E}-6$ std. cc He/sec.

$3 \times 10 \mathrm{E}-6$ std. $\mathrm{cc} \mathrm{He} / \mathrm{sec}$.

Wires emersed in LN2 6" from the epoxy-

initial

5 x 10E-6 std. cc He/sec.

cold:

0-10min. $\quad 3 \times 10 \mathrm{E}-6 \mathrm{std} . \mathrm{cc} \mathrm{He} / \mathrm{sec}$.

Gradual increase to $\quad 4 \times 10 \mathrm{E}-6 \mathrm{std} . \mathrm{cc} \mathrm{He} / \mathrm{sec}$.

LN2 3" from the epoxy-

initial

$2.5 \times 10 \mathrm{E}-6$ std. $\mathrm{cc} \mathrm{He} / \mathrm{sec}$.

$0-15 \mathrm{~min}$. down to

$2 \times 10 \mathrm{E}-6$ std. cc He/sec.

After the test the warm leakage rate was-

$6 \times 10 \mathrm{E}-6$ std. $\mathrm{cc} \mathrm{He} / \mathrm{sec}$.

The next morning-

6.6 x 10E-6 std. $\mathrm{cc} \mathrm{He} / \mathrm{sec}$.

The wires were cooled again 6" from the epoxy and the following leakage rates were observed.

warm

cold

warm-up

One week later -

After welding-
6.6 x 10E-6 std. cc He/sec. $3 \times 10 \mathrm{E}-6$ std. $\mathrm{cc} \mathrm{He} / \mathrm{sec}$.

$3.4 \times 10 \mathrm{E}-6 \mathrm{std} . \mathrm{cc} \mathrm{He} / \mathrm{sec}$.

5 x 10E-6 std. cc He/sec.

5.6 x $10 \mathrm{E}-6$ std. cc He/sec.

$5.8 \times 10 \mathrm{E}-6$ std. $\mathrm{cc} \mathrm{He} / \mathrm{sec}$.

$6.8 \times 10 \mathrm{E}-6 \mathrm{std} . \mathrm{cc} \mathrm{He} / \mathrm{sec}$.

$7.5 \times 10 \mathrm{E}-6 \mathrm{std} . \mathrm{cc} \mathrm{He} / \mathrm{sec}$.

$7.1 \times 10 \mathrm{E}-6 \mathrm{std}$. cc He/sec.

$2 \times 10 \mathrm{E}-7$ std. $\mathrm{cc} \mathrm{He} / \mathrm{sec}$.

$5 \times 10 \mathrm{E}-6 \mathrm{std} . \mathrm{cc} \mathrm{He} / \mathrm{sec}$.

NOTE: Multiply values by .374 for std. cc air/sec. 


\section{Coaxial Cable Feed through}

Next, the feedthrough was pressure tested to 1500 psig with no obvious signs of leakage or physical damage. Following pressure testing we machined the feedthrough in half. The discernible details appear in the cartoon below.

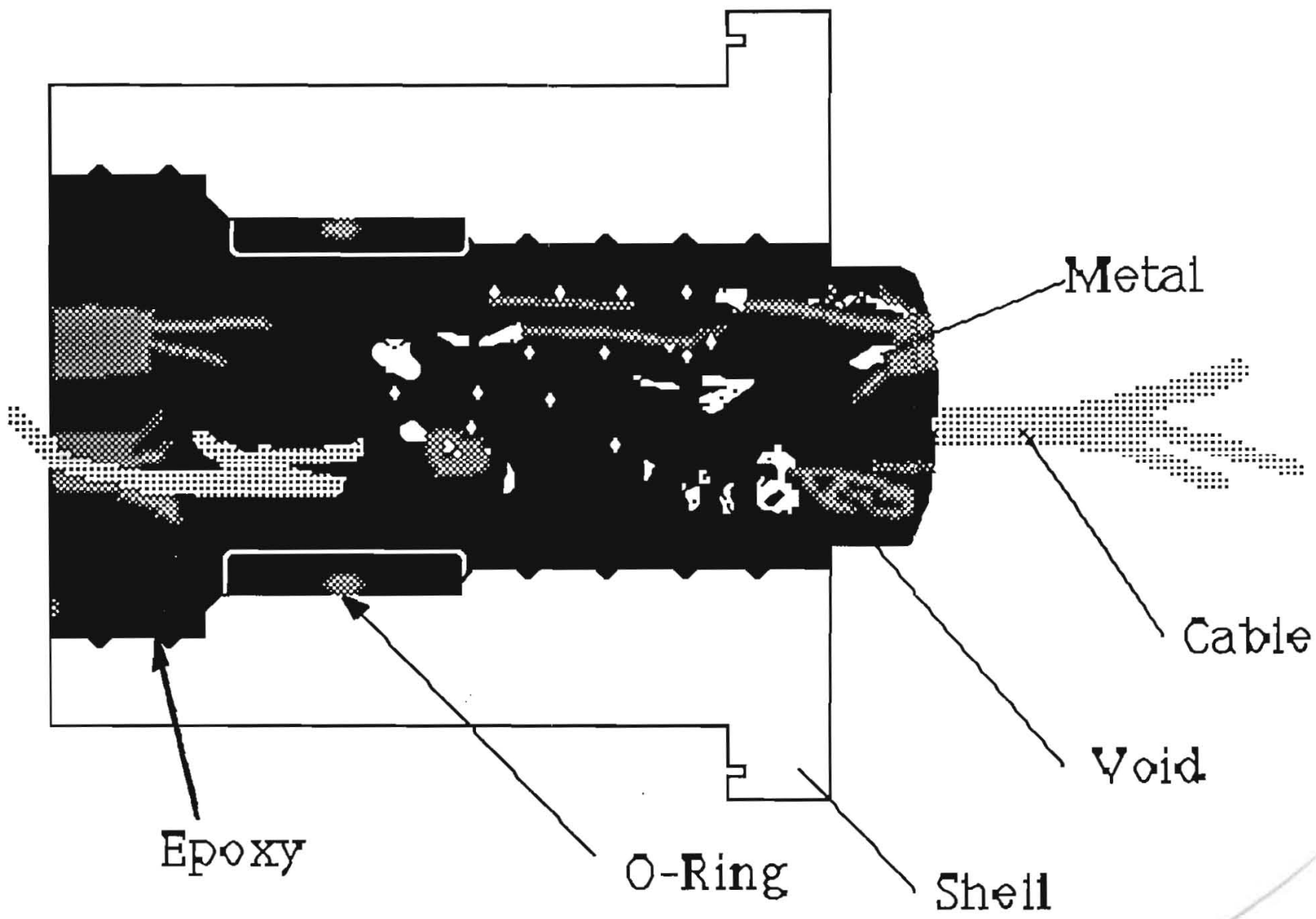




\section{Coaxial Cable Feedthrough}

\section{Conclusions}

This feedthroughs leakage rate after cold cycling, although not perfect, was not horrible. This and the results from pressure testing leave no reason for this feedthrough not to be used in the instrumentation box.

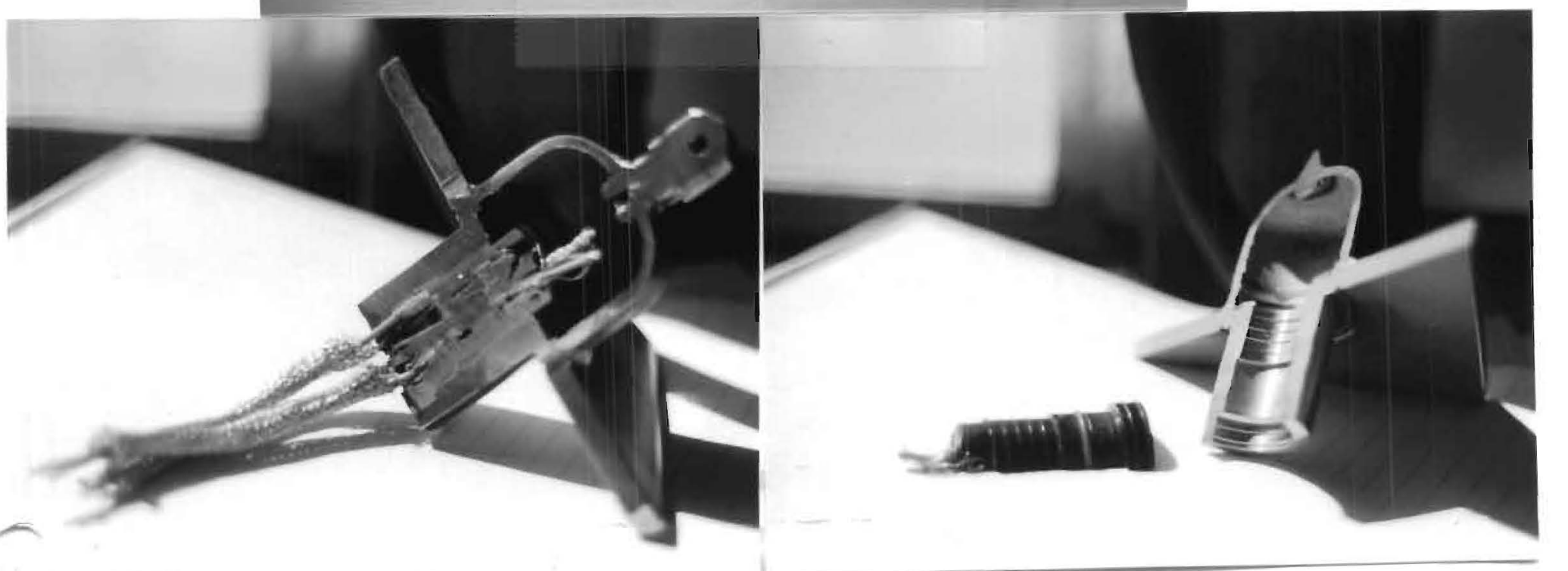

